\title{
ANALYSIS OF COUPLING IMPEDANCE BENCH MEASUREMENTS USING BETHE'S DIFFRACTION THEORY*
}

\author{
S. De Santis, LBNL, Berkeley, California.
}

\section{Abstract}

In this paper we study the validity of coupling impedance bench measurements, comparing two of the most commonly used formulas to the result obtained applying a modified version of Bethe's theory of diffraction to a lumped impedance in a coaxial beam pipe. The equations found provide a quantitative expression for the influence of the wire thickness used in the measurement of the real and imaginary part of the longitudinal impedance. The precision achievable in an actual measurement is therefore discussed. The method presented can also be applied in the presence of distributed impedances as well as to the analysis of transverse impedance measurements.

\section{INTRODUCTION}

Bethe's diffraction theory, in its modified version [1], has been successfully used to analytically calculate the coupling impedance of different structures that can be found in an accelerator vacuum chamber [2-5].

More recently, several papers have been dedicated to the theory of coupling impedance bench measurements, in particular regarding the classic coaxial wire method [6-7].

In this paper we use Bethe's theory to calculate the longitudinal impedance of a small aperture on a coaxial beam pipe, as it would be ideally measured in a coaxial wire set-up. The analytical formula obtained is compared to the formula derived in [2], which has been checked against MAFIA simulations and other semi-analytical methods. This comparison gives some insight on the influence of the wire on the measurement and on the differences between the various formulas used to relate the measured scattering parameters to the actual impedance.
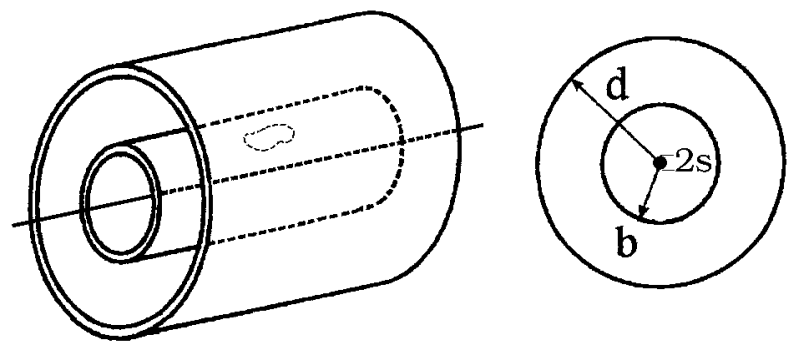

Figure 1: Relevant geometry

\section{MEASURED IMPEDANCE}

The longitudinal impedance of a small (with respect to the wavelength) aperture on a coaxial beam pipe as shown in Fig. 1 can be calculated from the measured $S_{21}$ parameter using the Hahn-Pedersen formula [8]:

$$
Z_{H P}=2 Z_{c} \frac{1-S_{21}^{D U T} / S_{21}^{R E F}}{S_{21}^{D U T} / S_{21}^{R E F}}
$$

where $Z_{c}$ is the characteristic impedance for a coaxial line equal to $60 \ln (b / s) \Omega$.

In Eq.(1) the $S_{21}$ measured for the device under test is normalized to that of a reference section of equal length. In the following we will always assume that the reference line has been calibrated out and will simply refer to $S_{21}$.

We also assume perfect matching in the measuring equipment and lossless materials and we will consider only TEM waves which is a rigorous treatment for frequencies below the $\mathrm{TE}_{1,1}$ mode cut-off, above which this measurement technique is not accurate anyway.

\subsection{Measured Impedance Calculation}

In the absence of the coupling aperture, the incident field $\left(E_{0 r}, H_{O \varphi}\right)$ is of course confined to the inner coaxial line and travels the length of the component experiencing only a phase delay. When this delay is taken into account by normalizing with the reference section, $S_{2 l}=1$ and the impedance is zero, as expected.

The presence of the aperture generates forward and backward scattered waves travelling in both coaxial regions. From the scattering matrix definition we can write:

$$
S_{21}=\frac{H_{0 \varphi}+H_{i \varphi}^{+}}{H_{0 \varphi}}=\frac{E_{0 r}+E_{i r}^{+}}{E_{0 r}}
$$

where $\left(E_{i r}^{+}, H_{i \varphi}^{+}\right)$is the forward wave in the inner region.

This wave can be expressed as:

$$
\begin{aligned}
E_{i r}^{+} & =c_{i}^{+} e_{i r} e^{-j k_{0} z} \theta(z) \\
H_{i \varphi}^{+} & =c_{i}^{+} h_{i \varphi} e^{-j k_{0} z} \theta(z)
\end{aligned}
$$

where $k_{0}=2 \pi / \lambda$ is the wavenumber, $\theta(z)$ is the Heaviside function, $Z_{0}=377 \Omega$ is the vacuum impedance, $e_{i r}$ and $h_{i \varphi}$ are the TEM modal function in the inner coax

$$
\begin{aligned}
e_{i r} & =\sqrt{\frac{Z_{0}}{2 \pi}} \frac{1}{\sqrt{\ln (b / s)}} \frac{1}{r} \\
h_{i \varphi} & =\frac{1}{\sqrt{2 \pi Z_{0}}} \frac{1}{\sqrt{\ln (b / s)}} \frac{1}{r}
\end{aligned}
$$

and the excitation constant $c_{i}^{+}$depends on the equivalent dipole moments of the aperture $M_{\varphi}$ and $P_{r}$ :

$$
c_{i}^{+}=-\frac{j \omega}{2}\left(\mu h_{i \varphi} M_{\varphi}+e_{i r} P_{r}\right)_{r=b}
$$

The equivalent dipole moments, in turn, depend on the aperture polarizabilities $\alpha_{e}$ and $\alpha_{m \perp}$ and on the incident and scattered fields:

\footnotetext{
*This work was supported by the U.S. Department of Energy under Contract No. DE-AC03-76SF00098.
} 


$$
\begin{gathered}
M_{\varphi}=\alpha_{m \perp}\left(H_{0 \varphi}+H_{i \varphi}-H_{o \varphi}\right)_{r=b} \\
P_{r}=\varepsilon \alpha_{e}\left(E_{0 r}+E_{i r}-E_{o \varphi}\right)_{r=b}
\end{gathered}
$$

where $\left(E_{o r}, H_{o \varphi}\right)$ is the scattered field in the outer coaxial region for which equations analogous to Eqs.(3-5) are valid, if we just replace $\ln (b / s)$ with $\ln (d / b)$ and the subscript $i$ with $o$.

From these equations we calculate the dipole moments expressions:

$$
M_{\varphi}=\frac{\alpha_{m \perp} H_{0 \varphi}}{1+j \frac{\alpha_{m \perp} k_{0}}{4 \pi b^{2} \Lambda}} \text { and } P_{r}=\frac{\varepsilon \alpha_{e} E_{0 r}}{1+j \frac{\alpha_{e} k_{0}}{4 \pi b^{2} \Lambda}}
$$

where

$$
\Lambda=\frac{\ln (d / b) \ln (b / s)}{\ln (d / b)+\ln (b / s)}
$$

It is worth noticing that the expression for the dipole moments obtained when directly calculating the impedance (i.e. with the wire removed) differs from Eq.(7) only in that $\Lambda=\ln (d / b)$ which is the limit for $s \rightarrow 0$ of Eq.(8). As it is already known from practice, impedances are best measured with the thinnest possible wire.

Replacing Eq.(7) in Eq.(5) we obtain the expression for the coefficient of the forward scattered wave in the

$$
c_{i}^{+}=\frac{-\frac{j \omega}{2}\left(\mu h_{i \varphi} H_{0 \varphi}\right)_{r=b}\left(\alpha_{e}+\alpha_{m \perp}+j \frac{2 \alpha_{e} \alpha_{m \perp} k_{0}}{4 \pi b^{2} \Lambda}\right)}{1-\frac{\alpha_{e} \alpha_{m \perp} k_{0}^{2}}{16 \pi^{2} b^{4} \Lambda^{2}}+j k_{0} \frac{\alpha_{e}+\alpha_{m \perp}}{4 \pi b^{2} \Lambda}}
$$

and from Eqs.(2) and (3) the scattering coefficient is therefore:

$$
S_{21}=1-j \frac{\frac{k_{0}}{4 \pi b^{2} \ln (b / s)}\left(\alpha_{e}+\alpha_{m \perp}+j \frac{2 \alpha_{e} \alpha_{m \perp} k_{0}}{4 \pi b^{2} \Lambda}\right)}{1-\frac{\alpha_{e} \alpha_{m \perp} k_{0}^{2}}{16 \pi^{2} b^{4} \Lambda^{2}}+j k_{0} \frac{\alpha_{e}+\alpha_{m \perp}}{4 \pi b^{2} \Lambda}}
$$

Replacing Eq.(10) in Eq.(1), we obtain:

$$
Z_{H P}=\frac{j k_{0} Z_{0}}{4 \pi^{2} b^{2}} \frac{\alpha_{e}+\alpha_{m \perp}+j \frac{2 \alpha_{e} \alpha_{m \perp} k_{0}}{4 \pi b^{2} \Lambda}}{1-\frac{\alpha_{e} \alpha_{m \perp} k_{0}^{2}}{16 \pi^{2} b^{4} \Lambda^{2}}+j k_{0} \frac{\alpha_{e}+\alpha_{m \perp}}{4 \pi b^{2} \Lambda}}\left(1-\frac{j k_{0}}{4 \pi b^{2} \ln (b / s)} \frac{\alpha_{e}+\alpha_{m \perp}+j \frac{2 \alpha_{e} \alpha_{m \perp} k_{0}}{4 \pi b^{2} \Lambda}}{1-\frac{\alpha_{e} \alpha_{m \perp} k_{0}^{2}}{16 \pi^{2} b^{4} \Lambda^{2}}+j k_{0} \frac{\alpha_{e}+\alpha_{m \perp}}{4 \pi b^{2} \Lambda}}\right)^{-1}
$$

\section{COMPARISON WITH THEORY}

An analytical formula for the longitudinal impedance of a small aperture at low frequencies has been derived in [2] using the modified Bethe's diffraction theory:

$$
Z_{/ /} \approx \frac{k_{0} Z_{0}}{4 \pi^{2} b^{2}}\left[j\left(\alpha_{e}+\alpha_{m \perp}\right)+\frac{k_{0}\left(\alpha_{e}^{2}+\alpha_{m \perp}^{2}\right)}{4 \pi b^{2} \ln (d / b)}\right]
$$

We can compare Eq.(13) to Eq.(11) simplified for small aperture dimensions and low frequency. To this end we keep in mind that the polarizabilities are proportional to the aperture average radius cubed and that $k_{0}$ is proportional to the frequency.

Therefore, disregarding higher order terms in $\alpha_{e}, \alpha_{m \perp}$ and $k_{0}$ Eq.(12) becomes:

$$
\begin{aligned}
& Z_{H P} \approx \frac{k_{0} Z_{0}}{4 \pi^{2} b^{2}}\left\{j\left(\alpha_{e}+\alpha_{m \perp}\right)+\right. \\
& \left.+\frac{k_{0}}{4 \pi b^{2} \ln (d / b)}\left[\left(\alpha_{e}+\alpha_{m \perp}\right)^{2}-2 \alpha_{e} \alpha_{m \perp} \frac{\ln (d / s)}{\ln (b / s)}\right]\right\}
\end{aligned}
$$

It is also interesting to compare Eqs.(13) and (14) to the impedance obtained from the measured $S_{21}$ using Sands and Rees expression [9]:

$$
Z_{S R}=2 Z_{c}\left(1-S_{21}\right)
$$

which, substituting Eq.(10) and simplifying for small aperture dimensions and low frequencies, becomes:

$$
\begin{aligned}
Z_{S R} \approx & \frac{k_{0} Z_{0}}{4 \pi^{2} b^{2}}\left\{j\left(\alpha_{e}+\alpha_{m \perp}\right)+\right. \\
& \left.+\frac{k_{0}}{4 \pi b^{2} \ln (d / b)}\left[\left(\alpha_{e}^{2}+\alpha_{m \perp}^{2}\right) \frac{\ln (d / s)}{\ln (b / s)}\right]\right\}
\end{aligned}
$$

\subsection{Imaginary Impedance}

We can see immediately that the imaginary part of the impedance, as calculated in Eqs. $(13,14)$ and $(16)$ is exactly the same:

$$
\operatorname{Im}\left(Z_{/ /}\right) \approx j \frac{k_{0} Z_{0}}{4 \pi^{2} b^{2}}\left(\alpha_{e}+\alpha_{m \perp}\right)
$$

This is not surprising since the imaginary impedance is dominated by the reactive energy stored in the modes below cut-off (i.e. non propagating) near the aperture. These modes are, of course, not much influenced by the presence of the wire.

It is worth pointing out that, apparently, Eq.(17) is totally independent from the wire radius $s$. This is not so as, if $s$ should increase to become comparable with $b$, the aperture polarizabilities would be modified.

\subsection{Real Impedance}

In this case Eqs. $(13,14)$ and $(16)$ coincide only in the limit $s \rightarrow 0$, as already stated above:

$$
\begin{gathered}
\operatorname{Re}\left(Z_{/ /}\right) \approx \frac{k_{0}^{2} Z_{0}}{16 \pi^{3} b^{4} \ln (d / b)}\left(\alpha_{e}^{2}+\alpha_{m \perp}^{2}\right), \\
\operatorname{Re}\left(Z_{S R}\right) \approx \frac{k_{0}^{2} Z_{0}}{16 \pi^{3} b^{4} \ln (d / b)}\left(\alpha_{e}^{2}+\alpha_{m \perp}^{2}\right) \frac{\ln (d / s)}{\ln (b / s)}
\end{gathered}
$$

and

$$
\begin{aligned}
\operatorname{Re}\left(Z_{H P}\right) \approx & \frac{k_{0}^{2} Z_{0}}{16 \pi^{3} b^{4} \ln (d / b)}\left[\left(\alpha_{e}+\alpha_{m \perp}\right)^{2}+\right. \\
& \left.-2 \alpha_{e} \alpha_{m \perp} \frac{\ln (d / s)}{\ln (b / s)}\right]
\end{aligned}
$$

To further analyze the difference between the various formulas we define an "error" function as follows: 


$$
E_{* *}=\frac{\operatorname{Re}\left(Z_{* *}\right)-\operatorname{Re}\left(Z_{/ /}\right)}{\operatorname{Re}\left(Z_{/ /}\right)}
$$

where $Z_{* *}$ can be either one of the measured impedances $Z_{H P}$ and $Z_{S R}$

We find

$$
E_{S R}=\frac{\ln (d / s)}{\ln (b / s)}-1
$$

and

$$
E_{H P}=\frac{2 \alpha_{e} \alpha_{m \perp}\left(1-\frac{\ln (d / s)}{\ln (b / s)}\right)}{\alpha_{e}^{2}+\alpha_{m \perp}^{2}}
$$

A first fact to notice is that Eq.(22) does not depend on the aperture polarizabilities, which are generally unknown. This means that, in principle, it would be possible to reconstruct the theoretical value of the longitudinal impedance from its value measured using Sands and Rees formula.

As Eq.(23) is regarded, let's remind that transverse magnetic and electric polarizabilities always have opposite sign and write $\left|\alpha_{m \perp}\right|=\gamma\left|\alpha_{e}\right|$. We have:

$$
E_{H P}=\frac{2 \gamma}{1+\gamma^{2}} E_{S R}
$$

The function multiplying $E_{S R}$ reaches its maximum value of 1 for $\gamma=1$ and decreases monotonically to zero elsewhere. $\gamma=1$ corresponds to the case of a very long rectangular or rounded-end slot.

This means that $E_{H P} \leq E_{S R}$, i.e. the impedance measured using the Hahn-Pedersen formula is always closer to the theoretical value than if the Sands-Rees formula is used. As an example, for a circular hole $\gamma=2$ and so $E_{H P}=0.8 E_{S R}$.

This is also consistent with the fact that the Sands-Rees formula can be derived from the Hahn-Pedersen one as the latter approximation for small values of the impedance [8].

\section{CONCLUSIONS}

In this paper we used a modification of Bethe's diffraction theory to calculate the impedance of an aperture in a coaxial beam pipe as it would be measured using the classic coaxial wire technique. This result has been compared to the impedance value obtained applying directly the diffraction theory.

The imaginary part of the impedance is not affected, in first approximation, by the wire presence and the HahnPedersen and the Sands-Rees formulas give the same result as the direct calculation.

For the real impedance, the Hahn-Pedersen formula gives an impedance always closer to the theoretical value than the Sands-Rees one. The difference between theoretical impedance and as calculated by Sands-Rees does not depend on the aperture polarizabilities so that it is possible to write a general formula that allows to obtain the theoretical impedance value from the measured one:

$$
Z_{/ /}=\operatorname{Re}\left(Z_{S R}\right) \frac{\ln (b / s)}{\ln (d / s)}+j \operatorname{Im}\left(Z_{S R}\right)
$$

The procedure presented in this paper can also be extended to the study of the transverse impedance and used for structures more complex than this simple example.

\section{ACKNOWLEDGEMENTS}

The author wishes to thank Harald Hahn for suggesting the application of the modified diffraction theory to the impedance measurement problem.

\section{REFERENCES}

[1] R.E. Collin, Field Theory of Guided Waves, $2^{\text {nd }}$ ed. IEEE Press, New York, 1991.

[2] S. De Santis, L. Palumbo, et al. Phys. Rev. E 54, 800 (1996).

[3] S. De Santis and L. Palumbo Phys. Rev. E 55, 2052 (1997).

[4] S. De Santis, L. Palumbo, et al. Phys. Rev. E 56, 5990 (1997).

[5] S. De Santis, L. Palumbo, et al. Phys. Rev. E 58, 6565 (1998).

[6] A. Argan, L. Palumbo, et al. Proc. of PAC 1999, 1599, New York (1999).

[7] H. Hahn, Phys. Rev. ST - Accel. Beams 3, 122001 (2000).

[8] H. Hahn and F. Pedersen, BNL 50870 (1978).

[9] M. Sands and J. Rees, SLAC report PEP-95 (1974). 